\title{
Near-crack-tip measurements within an Arlequin modeling for the numerical evaluation of far-field quantities of interest
}

\author{
Olivier Jamond, Guillaume Puel, Laurent Humbert and Hachmi Ben Dhia
}

Although numerical models are gradually improving, a relevant description of local phenomena such as fracture or crack propagation is still out of reach. As a result, the scope of these models is often restricted as far as the question of reliable prediction is addressed. This is the case even if the quantity of interest to be evaluated does not lie in the vicinity of the local phenomenon.

As a possible solution to this issue, we propose here to use the Arlequin framework [BEN98, BEN05] to mix experimental data measured in the vicinity of the local phenomenon with a global numerical model suited for the prediction of a specific quantity of interest. Let us recall here that the Arlequin method is a versatile numerical approach which, as shown in Figure 1, allows for the superposition and coupling of different models on a specific zone, typically a surface or a volume.

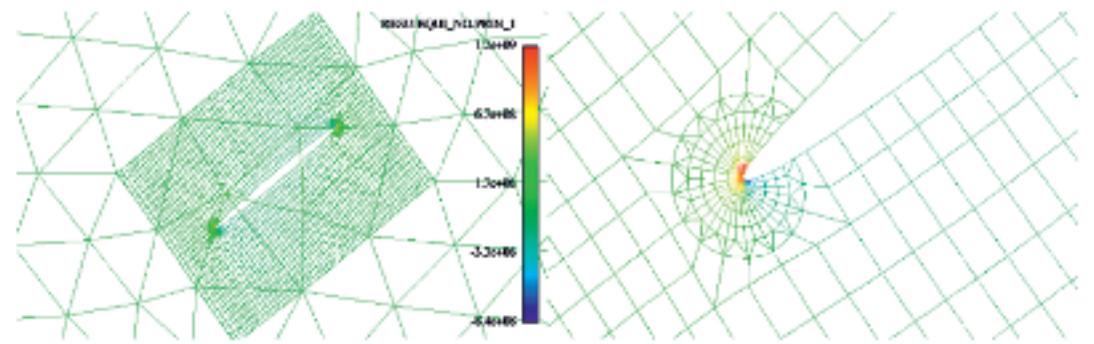

Figure 1. Illustration of the Arlequin framework.

This strategy is applied to a compact tension (CT) specimen made of epoxy and PMMA, which is subjected to cyclic loading. A chevron notch is typically machined to initiate the crack, whereas a hole is drilled far from the introduced crack tip. The purpose of the hole is to introduce a stress concentration phenomenon constituting the quantity of interest, whose value is influenced by the crack propagation. The lateral surfaces of the specimen are prepared with white painting and sprayed with a black aerosol resulting in a random structured macroscopic pattern for displacement field observations. The in-plane displacement field in the vicinity of the propagating crack tip is evaluated from the digital image correlation technique with the software Correli ${ }^{\mathrm{Q} 4}$ developed by F. Hild and coworkers [HIL08]. The displacement is typically evaluated by minimizing a so-called objective function that results from the comparison of pairs of digital images acquired during the cyclic loading. 
The Arlequin framework will then be used to couple the experimental displacement field in an annular zone around the propagating crack tip with a simple numerical elastic model, which is well suited for the evaluation of the quantity of interest along the hole. Instead of applying the experimental displacements as boundary conditions for the numerical model, the Arlequin framework allows to couple both the measurements and the model in a specific surface, which should improve the resolution of the numerical model.

The strategy will be validated by means of a second CCD camera permetting to measure the strain field in the vicinity of the hole, in order to estimate the quality of the prediction

[BEN98] Hachmi Ben Dhia. Multiscale mechanical problems: the Arlequin method. Comptes-rendus de l'Académie des Sciences Série II Fascicule B - Mécanique Physique Astronomie 326(12), pp. 899904, 1998.

[BEN05] Hachmi Ben Dhia and Guillaume Rateau. The Arlequin method as a flexible engineering design tool. International Journal for Numerical Methods in Engineering 62(11), pp. 1442-1462, 2005 [HIL08] François Hild and Stéphane Roux. Correli ${ }^{\mathrm{Q}}$ - A software for "Finite-element" Displacement Field Measurements by digital Image Correlation. Internal report. April 2008 